\title{
On Subadditive Functions Bounded Above on a "Large" Set
}

Nicholas H. Bingham, Eliza Jabłońska, Wojciech Jabłoński, and Adam J. Ostaszewski

In memoriam Harry I. Miller (1939-2018)

\begin{abstract}
It is well known that boundedness of a subadditive function need not imply its continuity. Here we prove that each subadditive function $f: X \rightarrow \mathbb{R}$ bounded above on a shift-compact (non-Haar-null, non-Haar-meagre) set is locally bounded at each point of the domain. Our results refer to results from Kuczma's book (An Introduction to the theory of functional equations and inequalities. Cauchy's equation and Jensen's inequality, 2nd edn, Birkhäuser Verlag, Basel, 2009, Chapter 16) and papers by Bingham and Ostaszewski [Proc Am Math Soc 136(12):4257-4266, 2008, Aequationes Math 78(3):257-270, 2009, Dissert Math 472:138pp., 2010, Indag Math (N.S.) 29:687-713, 2018, Aequationes Math 93(2):351-369, 2019).
\end{abstract}

Mathematics Subject Classification. Primary 39B62; Secondary 28C10, 18B30, 54E52.

Keywords. Shift-compact set, null-finite set, Haar-null set, Haar-meagre set, subadditive function, local boundedness at a point, WNT-function.

\section{Motivation and Auxiliary Results}

Let $X$ be an Abelian topological group. A function $f: X \rightarrow \mathbb{R}$ is subadditive if $^{1}$

$$
f(x+y) \leq f(x)+f(y) \quad \text { for every } x, y \in X .
$$

\footnotetext{
${ }^{1}$ In the paper we do not admit infinite values of $f$. For more information on infinite-valued subadditive functions see [31, Chapter 16].
} 
A function $f: X \rightarrow \mathbb{R}$ which is subadditive and also satisfies

$$
f(n x)=n f(x) \quad \text { for every } x \in X, n \in \mathbb{N}
$$

is called sublinear.

Subadditive and sublinear functions play a fundamental role in mathematics and so have attracted the interest of many authors (see e.g. [3,5,20,27, 31,33,34,42]). Examples of subadditive functions include norms, seminorms, and the function $\mathbb{R} \ni x \mapsto \sqrt{|x|} \in \mathbb{R}$ (see e.g. [27, Theorem 7.2.5]). A classical result concerning subadditive functions is.

Theorem 1.1 ([31, Theorem 16.2.2]). If a subadditive function $f: \mathbb{R}^{N} \rightarrow \mathbb{R}$ is locally bounded above at some point, then $f$ is locally bounded at each point of $\mathbb{R}^{N}$.

Note that local boundedness of a subadditive function does not imply its continuity; any function having values in the interval $[a, 2 a]$, with $a>0$, is subadditive.

A result stronger than Theorem 1.1 is the following.

Theorem 1.2 ([31, Theorem 16.2.4]). If a subadditive function $f: \mathbb{R}^{N} \rightarrow \mathbb{R}$ is bounded above on a set $T \subset \mathbb{R}^{N}$ and such that its $k$-fold sum $\sum_{i=1}^{k} T$ has positive inner Lebesgue measure or is non-meagre for some $k \in \mathbb{N}$, then $f$ is locally bounded at each point of $\mathbb{R}^{N}$.

Below we generalize the two theorems above using the notion of a shiftcompact set (see [39, III.2], [26, 5.1]).

Definition 1.3. In an Abelian topological group $X$, a set $A \subset X$ is called shiftcompact if for every sequence $\left(x_{n}\right)_{n \in \mathbb{N}}$ tending to 0 in $X$ there exists $x \in X$ such that the set $\left\{n \in \mathbb{N}: x+x_{n} \in A\right\}$ is infinite.

Shift-compact sets were used in the context of subadditive functions for the first time by Bingham and Ostaszewski in [9]. In the Euclidean context of [6] a shift-compact set was earlier called subuniversal (following Kestelman $[30]$ ), and also null-shift-compact (in [11] in the special context of $\mathbb{R}$ ). The underlying group action is studied in [37].

The notion of a shift-compact set is directly equivalent to the notion of a non-null-finite set. The definition of null-finite sets was introduced by Banakh and Jabłońska in [2] (see also [32]). Using [2, Proposition 2.2] we can easily explain the name "shift-compact": a set is shift-compact if and only if infinitely many points of this set belong to a translation of any infinite compact set. It is clear that non-empty open sets are shift-compact. Moreover, in view of [2, Theorem $3.1(2)]$, countable sets in non-discrete metric groups are not shift-compact. The result of Kwela [32, Theorem 4.1], that the Cantor set is not shift-compact, seems to be of significant interest. 
To indicate the extent of shift-compact sets we need to recall two terms: Haar-null sets, as defined by Christensen in [16], and their topological analogue the Haar-meagre sets, as defined by Darji [23].

Definition 1.4. Let $X$ be a complete Abelian metric group. A set $A \subset X$ is called universally measurable ${ }^{2}$ if it is measurable with respect to each complete Borel probability measure on $X$. A universally measurable set $B \subset X$ is Haarnull if there exists a $\sigma$-additive probability Borel measure $\mu$ on $X$ such that $\mu(B+x)=0$ for all $x \in X$.

Christensen [16] proves that in each locally compact complete Abelian metric group the notion of a Haar-null set is equivalent to the notion of a set of Haar measure zero.

Definition 1.5. Let $X$ be a complete Abelian metric group. A set $A \subset X$ is called universally Baire ${ }^{3}$ if for each continuous function $f: K \rightarrow X$ mapping a compact metric space $K$ into $X$ the set $f^{-1}(A+x)$ has the Baire property for every $x \in X$. A universally Baire set $B \subset X$ is called Haar-meagre if there exists a continuous map $f: K \rightarrow X$ from a non-empty compact metric space $K$ such that the set $f^{-1}(B+x)$ is meagre in $K$ for every $x \in X$.

Darji [23] shows that in each locally compact complete Abelian metric group the notion of a Haar-meagre set is equivalent to the notion of a meagre set. However, in the non-locally compact case, there is only a one-sided inclusion: Haar--meagre sets are meagre, but the converse implication may fail.

Armed with these terms, we may now note (see [2, Theorems 5.1 and 6.1] and also [14, Theorem 3]) that

Theorem 1.6. In a complete Abelian metric group: ${ }^{4}$

(i) each universally Baire non-Haar-meagre set is shift-compact;

(ii) each universally measurable non-Haar-null set is shift-compact.

The converse of Theorem 1.6 does not hold: see [19, Theorem 12], [2, Example 7.1] and also [38].

It emerges that in non-locally compact complete Abelian metric groups there exist sets which are neither Haar-null nor Haar-meagre and with $k$-fold sums that are meagre for each $k \in \mathbb{N}$. An example is provided by the positive cone $C:=\left\{\left(x_{n}\right)_{n \in \mathbb{N}} \in c_{0}: x_{n} \geq 0\right.$ for each $\left.n \in \mathbb{N}\right\}$ in the space $c_{0}$ (of all real sequences tending to zero); this is neither Haar-meagre nor Haar-null, $C=\sum_{i=1}^{k} C$ for each $k \in \mathbb{N}$ and $C$ is nowhere dense in $X$ (see [28,35]). By the Steinhaus-Pettis-Piccard Theorem (see [40,41,43] or [31, Theorems 2.9.1, 3.7.1]), such a situation is not possible in the case of locally compact Abelian

\footnotetext{
${ }^{2}$ See e.g. [29, p. 227].

${ }^{3}$ See e.g. $[25]$.

${ }^{4}$ Here by a metric group we mean a group with an invariant metric; by the Birkhoff-Kakutani theorem (see e.g. [29, Theorem 9.1]) any metrizable topological group is a metric group.
} 
Polish groups, where the families of Haar-meagre sets and of meagre sets coincide, and likewise the families of Haar-null sets and of sets of Haar measure zero coincide. This motivates the following:

Question 1.7. For $X$ a complete Abelian metric group and $f: X \rightarrow \mathbb{R}$ a subadditive function, bounded above on a set $T \subset X$ with a $k$-fold sum $\sum_{i=1}^{k} T$ either universally Baire and non-Haar-meagre, or universally measurable and non-Haar-null, is $f$ locally bounded at each point of $X$ ?

Below we give an affirmative answer. Actually, we prove a more general result: we show that for $X$ an Abelian metric group, every subadditive function $f: X \rightarrow \mathbb{R}$ that is bounded above on a shift-compact subset of $X$ is necessarily locally bounded at each point of $X$.

Analogous results for additive functions as well as mid-point convex functions [i.e. functions satisfying

$$
f((x+y) / 2) \leq(f(x)+f(y)) / 2
$$

for every $x, y$ from the domain of $f]$ were obtained in [2, Theorems 9.1 and $11.1]$, and also in $[6, \S 7$ and Theorem 1$]$ in the case of $\mathbb{R}$ (cf. [9, $\S 10$ Convexity]), where the following two results were proved. (We use 'shift-compact' in place of 'non-null-finite' as in [1]; see $\S 1$.)

Theorem 1.8 ([2, Theorem 9.1]). Each additive functional $f: X \rightarrow \mathbb{R}$ on an Abelian metric group $X$ is bounded above on a shift-compact set in $X$ if and only if it is continuous.

Theorem 1.9 ([2, Theorem 11.1]). Each mid-point convex function $f: X \rightarrow \mathbb{R}$ defined on a real linear metric space $X$ is bounded above on a shift-compact set in $X$ if and only if it is continuous.

Since each sublinear function $f: X \rightarrow \mathbb{R}$ defined on a real linear space $X$ is necessarily mid-point convex (see [31, Lemma 16.1.11]), from Theorem 1.9 we obtain

Corollary 1.10. Each sublinear function $f: X \rightarrow \mathbb{R}$ defined on a real linear metric space $X$ is bounded above on a shift-compact set in $X$ if and only if it is continuous.

The result above in the case $X=\mathbb{R}$ was obtained by Bingham and Ostaszewski in [12, Proposition 5] (cf. [13, Theorem R] and [15, Proposition $5])$.

This is also why boundedness from above of subadditive functions on shift-compact sets seems to be all the more interesting.

Finally, we determine the relationship between local boundedness at some point, boundedness from above on a shift-compact set and property WNT, as proposed by Bingham and Ostaszewski in [5], in the class of functions $f: X \rightarrow \mathbb{R}$ defined on an Abelian topological group $X$. 


\section{Main Results}

First, let us recall some basic properties of subadditive functions.

Lemma 2.1 ([31, Lemmas 16.1.3, 16.1.4, 16.1.5]). Let $X$ be a group and $f$ : $X \rightarrow \mathbb{R}$ be a subadditive function. Then:

(i) $f(0) \geq 0$;

(ii) $f(-x) \geq-f(x)$ for each $x \in X$.

We are now ready to prove the main result, which in the case of $\mathbb{R}$, was obtained in [6, Theorem 2 (ii) and Remark].

Theorem 2.2. Let $X$ be an Abelian metric group and $f: X \rightarrow \mathbb{R}$ a subadditive function. If $f$ is bounded above on a set $T \subset X$ whose $k$-fold sum $\sum_{i=1}^{k} T$ is shift-compact for some $k \in \mathbb{N}$, then $f$ is locally bounded at each point of $X$.

In the proof of the above result we use classical methods from [31, Proof of Theorem 16.2] and from [6, Proof of Theorem 2 (ii)].

Proof. Suppose that $f$ is not locally bounded at the point $x_{0} \in X$. This means that there is a sequence $\left(x_{n}\right)_{n \in \mathbb{N}}$ with $x_{n} \rightarrow x_{0}$ and $\left|f\left(x_{n}\right)\right| \rightarrow \infty$. Then we may choose either a subsequence $\left(x_{n}^{\prime}\right)_{n \in \mathbb{N}}$ of $\left(x_{n}\right)_{n \in \mathbb{N}}$ with $f\left(x_{n}^{\prime}\right)>n$ for each $n \in \mathbb{N}$ or a subsequence $\left(x_{n}^{\prime \prime}\right)_{n \in \mathbb{N}}$ of $\left(x_{n}\right)_{n \in \mathbb{N}}$ with $f\left(x_{n}^{\prime \prime}\right)<-n$ for each $n \in \mathbb{N}$.

In the first case, put $y_{n}:=x_{n}^{\prime}$ for $n \in \mathbb{N}$ and $y_{0}:=x_{0}$. In the second case, take $y_{n}:=-x_{n}^{\prime \prime}$ for $n \in \mathbb{N}$ and $y_{0}:=-x_{0}$. By Lemma 2.1, $f\left(-x_{n}^{\prime \prime}\right) \geq$ $-f\left(x_{n}^{\prime \prime}\right)>n$ for each $n \in \mathbb{N}$ so, in both cases, there exists a sequence $y_{n} \rightarrow y_{0}$ such that $f\left(y_{n}\right)>n$ for $n \in \mathbb{N}$. Since $y_{n}-y_{0} \rightarrow 0$ and $\sum_{i=1}^{k} T \subset X$ is shiftcompact, there exists $z_{0} \in X$ such that the set $\mathbb{N}_{0}:=\left\{n \in \mathbb{N}: z_{0}+y_{n}-y_{0} \in\right.$ $\left.\sum_{i=1}^{k} T\right\}$ is infinite. Moreover, by hypothesis, there exists a constant $M \in \mathbb{R}$ with $f\left(\sum_{i=1}^{k} x_{i}\right) \leq \sum_{i=1}^{k} f\left(x_{i}\right) \leq k M$ for each $x_{1}, \ldots, x_{k} \in T$; so $f(x) \leq k M$ for each $x \in \sum_{i=1}^{k} T$. Thus, by Lemma 2.1,

$$
n<f\left(y_{n}\right) \leq f\left(y_{n}-y_{0}+z_{0}\right)+f\left(y_{0}-z_{0}\right) \leq k M+f\left(y_{0}-z_{0}\right)
$$

for each $n \in \mathbb{N}_{0}$, so $\mathbb{N}_{0}$ is finite, a contradiction.

Next we consider some applications of Theorem 2.2.

Since each non-empty open set is shift-compact, we obtain the following generalization of Theorem 1.1.

Corollary 2.3. If $X$ is an Abelian metric group and $f: X \rightarrow \mathbb{R}$ a subadditive function locally bounded above at some point, then $f$ is locally bounded at every point of $X$.

The above corollary is also obtained in the case of $\mathbb{R}$ in $[13$, Theorem $R]$ (cf. also [9, Lemma 4.3]), but the proof there relies only on group structure, as here. 
By Theorem 1.6, in a complete Abelian metric group each universally Baire non-Haar-meagre set, and also each universally measurable non-Haarnull set, is shift-compact. Thus we obtain the next result, generalizing to some extent Theorem 1.2.

Corollary 2.4. If $X$ is a complete Abelian metric group and $f: X \rightarrow \mathbb{R}$ a subadditive function bounded above on a set $T \subset X$ with some $k$-fold sum $\sum_{i=1}^{k} T$ either universally Baire and non-Haar-meagre, or universally measurable and non-Haar-null, then $f$ is locally bounded at each point of $X$.

\section{A Connection with Generic Subadditive Functions}

In 2008 Bingham and Ostaszewski [5] (see also [7,8]) introduced the notion of Weak No Trumps functions (called WNT-functions).

Definition 3.1. Let $f: X \rightarrow \mathbb{R}$ be defined on an Abelian metric group ${ }^{5}$ and $H^{k}:=f^{-1}(-k, k)$ for $k \in \mathbb{N}$. Call $f$ a WNT-function if for every convergent sequence $\left(u_{n}\right)_{n \in \mathbb{N}}$ in $X$ there exist $k \in \mathbb{N}$, an infinite set $\mathbb{M} \subset \mathbb{N}$ and $t \in X$ such that $\left\{t+u_{m}: m \in \mathbb{M}\right\} \subset H^{k}$.

The more basic No Trumps combinatorial principle, denoted NT, refers to a family of subsets of reals $\left\{T_{k}: k \in \mathbb{N}\right\}$ (see [7, Definition 2]). The function class WNT is one of a hierarchy introduced in [8] and is so named as it refers to the weakest condition in the hierarchy.

One readily observes the following.

Lemma 3.2. A function $f: X \rightarrow \mathbb{R}$ defined on an Abelian metric group $X$ is WNT if and only if for every sequence $\left(u_{n}\right)_{n \in \mathbb{N}}$ convergent to 0 in $X$ there exist $k \in \mathbb{N}$, an infinite set $\mathbb{M} \subset \mathbb{N}$ and $t \in X$ such that $\left\{t+u_{m}: m \in \mathbb{M}\right\} \subset H^{k}$.

Now let us present some connections between the WNT-property, boundedness on a shift-compact set, and local boundedness at a point.

Proposition 3.3. Let $f: X \rightarrow \mathbb{R}$ be defined on an Abelian metric group. Then the following implications hold:

(i) if $f$ is locally bounded at a point, then $f$ is bounded on a shift-compact set in $X$;

(ii) if $f$ is bounded on a shift-compact set in $X$, then $f$ is WNT.

Proof. (i) It is an easy consequence of the fact that open sets are shift-compact.

(ii) Assume that for some shift-compact set $D \subset X$ there exists $k \in \mathbb{N}$ such that $f(D) \subset(-k, k)$. Since $D$ is shift-compact, for each sequence $\left(x_{n}\right)_{n \in \mathbb{N}}$ tending to 0 in $X$ there are $x_{0} \in X$ and an infinite set $\mathbb{N}_{0} \subset \mathbb{N}$ such that $f\left(x_{n}+x_{0}\right) \in f(D) \subset(-k, k)$ for each $n \in \mathbb{N}_{0}$. Consequently, for each sequence $\left(x_{n}\right)_{n \in \mathbb{N}}$ convergent to 0 there are $k \in \mathbb{N}, x_{0} \in X$ and an infinite set $\mathbb{N}_{0} \subset \mathbb{N}$ such that $x_{n}+x_{0} \in f^{-1}(-k, k)$ for $n \in \mathbb{N}_{0}$. So $f$ is WNT.

\footnotetext{
${ }^{5}$ In fact, Bingham and Ostaszewski defined a WNT-function in the case $X=\mathbb{R}^{N}$.
} 
Note that the converse implication to (i) in Proposition 3.3 does not hold. Example 1. Define a function $g:[0,1) \rightarrow \mathbb{R}$ by

$$
g(x):= \begin{cases}(-1)^{n} n, & \text { for } x=m / n \in \mathbb{Q} \cap(0,1), \text { where } \operatorname{gcd}(m, n)=1, \\ 0, & \text { for } x \in\{0\} \cup[(0,1) \backslash \mathbb{Q}] .\end{cases}
$$

First we prove that $g$ is locally unbounded at each point of $[0,1)$. Indeed, for each $x \in[0,1)$ every open neighbourhood $U_{x} \subset[0,1)$ of $x$ contains infinitely many positive rational numbers, hence $\sup _{t \in U_{x}}|g(t)|=\infty$.

Next, define a function $f: \mathbb{R} \rightarrow \mathbb{R}$ as 1 -periodic extension of $g$. Clearly, $f$ is locally unbounded at each point of $\mathbb{R}$.

Moreover, by Theorem 1.6, the set $\mathbb{R} \backslash \mathbb{Q}$ is shift-compact (as it has positive Lebesgue measure) and $f(\mathbb{R} \backslash \mathbb{Q})=\{0\}$. Thus, according to Proposition 3.3 (ii), $f$ is WNT.

Note also that there exists a function $f: \mathbb{R} \rightarrow \mathbb{R}$ bounded above on a shift-compact set in $\mathbb{R}$ which is not WNT. It means that we are not able to weaken the assumption in Proposition 3.3 (ii).

Example 2. Let $f: \mathbb{R} \rightarrow \mathbb{R}$ be given by $f(x):=-|g(x)|$ for $x \in \mathbb{R}$ with a discontinuous additive function $g: \mathbb{R} \rightarrow \mathbb{R}$. Clearly then $f$ is bounded above on $\mathbb{R}$. We have to show yet that $f$ is not WNT.

Since $g$ is additive and discontinuous, its graph is dense in $\mathbb{R}^{2}$ (see $[31$, Theorem 12.1.2]). So, there exists a sequence $\left(u_{n}\right)_{n \in \mathbb{N}}$ convergent to 0 such that $\left|g\left(u_{n}\right)\right|>n$ for every $n \in \mathbb{N}$. Fix $k \in \mathbb{N}$ and $t \in \mathbb{R}$. Then

$$
\begin{aligned}
\left\{n \in \mathbb{N}:\left|f\left(t+u_{n}\right)\right|<k\right\} & =\left\{n \in \mathbb{N}:-k-g(t)<g\left(u_{n}\right)<k-g(t)\right\} \\
& \subset\left\{n \in \mathbb{N}:\left|g\left(u_{n}\right)\right|<\max \{|k-g(t)|,|k+g(t)|\}\right\} .
\end{aligned}
$$

Hence the set $\left\{n \in \mathbb{N}: t+u_{n} \in f^{-1}((-k, k))\right\}$ is finite, which means that $\left\{t+u_{n}: n \in \mathbb{M}\right\} \not \subset H^{k}$ for every infinite set $\mathbb{M} \subset \mathbb{N}$.

In view of Example 2 we see that Theorem 2.2 can not be derived from the following result obtained in [5].

Proposition 3.4 ([5, Proposition 1$])$. If $f: \mathbb{R}^{N} \rightarrow \mathbb{R}$ is a subadditive WNTfunction, then $f$ is locally bounded at each point of $\mathbb{R}^{N}$.

We have shown that generally, for every $f: X \rightarrow \mathbb{R}$ defined on an Abelian metric group,

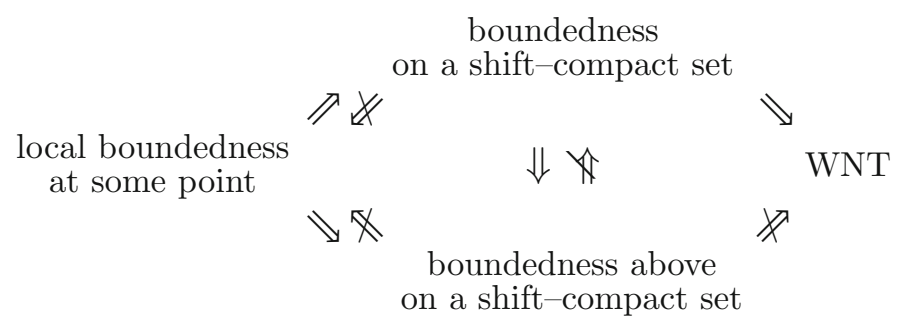


Question 3.5. Does every WNT function have to be bounded (above) on a shiftcompact set?

Nevertheless, combining Theorem 2.2 and Propositions 3.3 and 3.4, we deduce that the situation is completely different in the class of subadditive functions.

Corollary 3.6. Let $f: X \rightarrow \mathbb{R}$ be a subadditive function defined on an Abelian metric group. The following conditions are equivalent:

- $f$ is locally bounded at some point;

- $f$ is WNT;

- $f$ is bounded above on a shift-compact set;

- $f$ is bounded on a shift-compact set.

\section{Concluding Remarks}

4.1. This joint paper arose out of a newfound interest in shift-compactness. The concept was isolated a decade ago in establishing a common proof for the two known 'generic cases' of measure and category of the Uniform Convergence Theorem for regularly varying functions (for background see [4] and $[7])$. Most recently one of the current authors in collaboration initially with Banakh initiated the study of a common generalization of Christensen's notion of Haar-null sets and Darji's notion of Haar-meagre sets by replacing the relevant $\sigma$-ideals by other ideals $\mathcal{I}$ and working in the general context of Abelian metric groups. This yielded Haar- $\mathcal{I}$ sets, and led in particular to the notion of null-finite sets (corresponding to the ideal of finite subsets). A null-finite set is simply a non-shift-compact set and this explains the recent resurgence of interest in shift-compactness in metric groups, and the new results as in [38]. Results on subadditivity using shift-compactness have been studied in various publications in the last decade typically in Euclidean contexts, so it seemed natural to collect together the known results from the widely scattered literature, in the more natural contexts here of groups or linear spaces.

4.2. Use of large sets in the contexts of additivity, subadditivity, and convexity may be traced back to the work of Ger and Kuczma on 'test sets' (the GerKuczma classes of test sets $\mathfrak{A}, \mathfrak{B}, \mathfrak{C}[31$, Chapters 9,10]), the idea being that a property holding on a test set would automatically imply a related property globally, as in the well-known related case of automatic continuity (for which see Dales [1] and [21,22]). Such ideas were also pursued by Kominek on the basis of result by Jones (test set there being capable of spanning the reals, as with Hamel bases but not coincidental with these; for recent work on Hamel bases see e.g. $[17,18,24])$. An alternative approach to test sets was developed also in [10] with connections to uniformity results in the theory of regular variation. 
For further thematic uses of large sets (in connection with regularity, i.e. 'smoothness', properties of functions) see the extended arXiv version of this paper.

Postscript. With sadness we dedicate this paper to the memory of Harry I. Miller, friend and collaborator, recently passed away - a latter - day pioneer of shift-compactness (see [36]).

Open Access. This article is licensed under a Creative Commons Attribution 4.0 International License, which permits use, sharing, adaptation, distribution and reproduction in any medium or format, as long as you give appropriate credit to the original author(s) and the source, provide a link to the Creative Commons licence, and indicate if changes were made. The images or other third party material in this article are included in the article's Creative Commons licence, unless indicated otherwise in a credit line to the material. If material is not included in the article's Creative Commons licence and your intended use is not permitted by statutory regulation or exceeds the permitted use, you will need to obtain permission directly from the copyright holder. To view a copy of this licence, visit http://creativecommons. org/licenses/by/4.0/.

\section{References}

[1] Bachar, J.M., Bade, W.G., Curtis, Jr. P.C., Dales, H.G., Thomas, M.P.: Radical Banach Algebras and Automatic Continuity, Lect. Notes Math. 975, Springer (1981)

[2] Banakh, T., Jabłońska, E.: Null-finite sets in metric groups and their applications. Israel J. Math. 230, 361-386 (2019)

[3] Berz, E.: Sublinear functions on $\mathbb{R}$. Aequationes Math. 12, 200-206 (1975)

[4] Bingham, N.H., Goldie, C.M., Teugels, J.L.: Regular Variation, 2nd ed. Cambridge University Press, 1989 (1st ed. 1987)

[5] Bingham, N.H., Ostaszewski, A.J.: Generic subadditive functions. Proc. Am. Math. Soc. 136(12), 4257-4266 (2008)

[6] Bingham, N.H., Ostaszewski, A.J.: Automatic continuity: subadditivity, convexity, uniformity. Aequationes Math. 78(3), 257-270 (2009)

[7] Bingham, N.H., Ostaszewski, A.J.: Infinite combinatorics and the foundations of regular variation. J. Math. Anal. App. 360, 518-529 (2009)

[8] Bingham, N.H., Ostaszewski, A.J.: Beyond Lebesgue and Baire: Generic regular variation. Colloq. Math. 116, 119-138 (2009)

[9] Bingham, N.H., Ostaszewski, A.J.: Normed groups: Dichotomy and duality. Dissert. Math. 472, 138p (2010)

[10] Bingham, N.H., Ostaszewski, A.J.: Automatic continuity by analytic thinning. Proc. Am. Math. Soc. 138, 907-919 (2010)

[11] Bingham, N.H., Ostaszewski, A.J.: Dichotomy and infinite combinatorics: the theorems of Steinhaus and Ostrowski. Math. Proc. Camb. Philos. Soc. 150, 1-22 (2011) 
[12] Bingham, N.H., Ostaszewski, A.J.: Additivity, subadditivity and linearity: automatic continuity and quantifier weakening. Indag. Math. (N.S.) 29, 687-713 (2018)

[13] Bingham, N.H., Ostaszewski, A.J.: Variants of the Berz sublinearity theorem. Aequationes Math. 93(2), 351-369 (2019)

[14] Bingham, N.H., Ostaszewski, A.J.: The Steinhaus-Weil property: its converse, Solecki amenability and subcontinuity. arXiv:1607.00049v3 [math.GN]

[15] Bingham, N.H., Ostaszewski, A.J.: General regular variation, Popa groups and quantifier weakening. J. Math. Anal. Appl. 483(2), 123610 (2020)

[16] Christensen, J.P.R.: On sets of Haar measure zero in Abelian Polish groups. Israel J. Math. 13, 255-260 (1972)

[17] Ciesielski, K., Pawlikowski, J.: The Covering Property Axiom, CPA, Cambridge Tracts Math. 164, CUP (2004)

[18] Ciesielski, K., Pawlikowski, J.: Nice Hamel bases under the covering property axiom. Acta Math. Hungar. 105(3), 197-213 (2004)

[19] Ciesielski, K., Rosenblatt, J.: Restricted continuity and a theorem of Luzin. Colloq. Math. 135, 211-225 (2014)

[20] Cooper, R.: The converse of the Cauchy-Hölder inequality and the solution of the inequality $g(x+y) \leq g(x)+g(y)$. Proc. Lond. Math. Soc. 26, 415-432 (1927)

[21] Dales, H.G.: Automatic continuity: a survey. Bull. Lond. Math. Soc. 10(2), 129183 (1978)

[22] Dales, H.G.: Banach Algebras and Automatic Continuity. London Mathematical Society Monographs, vol. 24. Clarendon Press, Oxford (2000)

[23] Darji, U.B.: On Haar meager sets. Topol. Appl. 160, 2396-2400 (2013)

[24] Dorais, F., Filipów, R., Natkaniec, T.: On some properties of Hamel bases and their applications to Marczewski measurable functions. Open Math. 11(3), 487$508(2013)$

[25] Feng, Q., Magidor, M., Woodin, H.: Universally Baire sets of reals. In: Judah, H., Just, W., Woodin, H. (eds) Set Theory of the Continuum, pp. 203-242, Math. Sci. Res. Inst. Publ. 26, Springer, New York (1992)

[26] Heyer, H.: Probability Measures on Locally Compact Groups, Ergebnisse Math. Grenzgebiete 94, Springer (1977)

[27] Hille, E., Phillips, R.S.: Functional Analysis and Semigroups. American Mathematical Society Colloquium Publications XXXI. A.M.S, Providence (1957)

[28] Jabłońska, E.: Some analogies between Haar meager sets and Haar null sets in Abelian Polish groups. J. Math. Anal. Appl. 421, 1479-1486 (2015)

[29] Kechris, A.S.: Classical Descriptive Set Theory. Springer, New York (1995)

[30] Kestelman, H.: The convergent sequence belonging to a set. J. Lond. Math. Soc. 22, 130-136 (1947)

[31] Kuczma, M.: An Introduction to the Theory of Functional Equations and Inequalities. Cauchy's Equation and Jensen's Inequality, 2nd edn. Birkhäuser Verlag, Basel (2009). edited by A. Gilányi

[32] Kwela, A.: Haar-smallest sets. Topol. Appl. 267, 106892 (2019) 
[33] Matkowski, J., Świątkowski, T.: On subadditive functions. Proc. Am. Math. Soc. 119, 187-197 (1993)

[34] Matkowski, J.: Subadditive periodic functions. Opuscula Math. 31, 75-96 (2011)

[35] Matoušková, E., Zajiček, L.: Second order differentiability and Lipschitz smooth points of convex functionals. Czechoslovak Math. J. 48, 617-640 (1998)

[36] Miller, H.I.: Generalization of a result of Borwein and Ditor. Proc. Am. Math. Soc. 105(4), 889-893 (1989)

[37] Miller, H.I., Ostaszewski, A.J.: Group action and shift-compactness. J. Math. Anal. Appl. 392(1), 23-39 (2012)

[38] Miller, H.I., Miller-Van Wieren, L., Ostaszewski, A.J.: Beyond Erdös-KunenMauldin: Singular Sets with shift-compactness properties. arXiv:1901.09654

[39] Parthasarathy, K.R.: Probability Measures on Metric Spaces. Academic Press, New York (1967)

[40] Pettis, B.J.: Remarks on a theorem of E. J. McShane. Proc. Am. Math. Soc. 2, 166-171 (1951)

[41] Piccard, S.: Sur les ensembles de distances des ensembles de points d'un espace Euclidien, Mem. Univ. Neuchâtel, vol. 13, Secrétariat Univ., Neuchâtel (1939)

[42] Rosenbaum, R.A.: Sub-additive functions. Duke Math. J. 17, 227-247 (1950)

[43] Steinhaus, H.: Sur les distances des points des ensembles de mesure positive. Fund. Math. 1, 99-104 (1920)

Nicholas H. Bingham

Department of Mathematics

Imperial College London

South Kensington Campus

London SW7 2AZ

UK

e-mail: n.bingham@imperial.ac.uk

Eliza Jabłońska and Wojciech Jabłoński

Department of Mathematics

Pedagogical University of Cracow

Podchorążych 2

30-084 Kraków

Poland

e-mail: eliza.jablonska@up.krakow.pl

e-mail: wojciech.jablonski@up.krakow.pl

Adam J. Ostaszewski

Mathematics Department

London School of Economics

Houghton Street

London WC2A 2AEA

UK

e-mail: a.j.ostaszewski@lse.ac.uk 
Received: March 18, 2019.

Accepted: March 13, 2020.

Publisher's Note Springer Nature remains neutral with regard to jurisdictional claims in published maps and institutional affiliations. 